\title{
Intelligent Control of the Steering for a Powered Wheelchair Using a Microcomputer
}

\author{
Malik Haddad $^{1}$, David Sanders ${ }^{1}$, Martin Langner ${ }^{2}$, Mohamad Thabet ${ }^{1}$, Alexander \\ Gegov $^{1}$ and Favour Ikwan ${ }^{1}$ \\ ${ }^{1}$ University of Portsmouth, Portsmouth PO1 3DJ, UK \\ ${ }^{2}$ Chailey Heritage Foundation, North Chailey, BN8 4E, UK \\ david.sanderseport.ac.uk
}

\begin{abstract}
The research presented in this paper describes a new architecture for controlling powered wheelchairs. A Raspberry Pi microcomputer is considered to assist in controlling direction. A Raspberry $\mathrm{Pi}$ is introduced between user input switches and powered wheelchair motors to create an intelligent Human Machine Interface (HCI). An electronic circuit is designed that consists of an ultrasonic sensor array and a set of control relays. The sensors delivered information about obstructions in the surrounding environment of the wheelchair. Python programming language was used to create a program that digitized the user switches output and assessed information provided by the ultrasonic sensor array. The program was installed on a Raspberry Pi and the Raspberry Pi controlled power delivered to the motors. Tests were conducted and results showed that the new system successfully assisted a wheelchair user in avoiding obstacles. The new architecture can be used to intelligently interface any input device or sensor system to powered wheelchair.
\end{abstract}

Keywords: Direction, Avoidance, Python, Wheelchair, Raspberry Pi, Steer.

\section{Introduction}

Research described here is part of a bigger research project to improve mobility and enhance the quality of life of disabled powered wheelchair users by increasing their self-confidence and reliance [1]. A new architecture is described that acquires steering inputs from users and intelligently combines them with sensor readings to assist a disabled user to drive safely.

The World Health Organization's (WHO) report on disability stated about $15 \%$ of world population were suffering of some type of disability and 2-4\% of them experience significant difficulties in mobility. These numbers were higher than previous WHO estimates due to population ageing, rapid spread of chronic diseases, as well as modern medical treatment improvement [2].

In many cases, people with disabilities struggle with daily manoeuvring tasks and can be dependent on helpers and carers for other daily activities [3]. Disabled users of powered wheelchairs may not have the required ability or sufficient mobility due to hand, finger, shoulder, arm or more widespread incapability, and may not have 
adequate lower limb strength to propel a manual wheelchair [4]. An intelligent driving system could ease their mobility problem and reduce their dependency on others.

A smart wheelchair is a combination of a powered wheelchair with an intelligent sensor system and computer algorithms needed to make immediate intelligent decisions for path planning and collision avoidance [3]. A driver supplies speed and direction using an input device, for example levers, switches or joysticks. A wheelchair then starts to move at the desired speed towards the chosen direction. A user can then make adjustments to avoid obstacles. The preferred direction is combined with sensor output [5-17] to help the drivers.

Many researchers investigated navigation and steering [18-22] and obstacles avoidance [23] has been considered using local sensors [24], but they have barely moved out of the laboratory to assist disabled wheelchair users. A new architecture is described here that uses sensors to effectively assist with driving. The new system quickly responds to objects and aims to prevent a user from driving in the direction of an obstacle.

Raspberry Pi microcomputers have often been seen as reliable powerful microcomputers with small physical size and low cost. Two models were launched in 2012: A and B. In 2014, Model B+ was launched with numerous enhancements based on users feedback [25]. The research conducted in this paper used Raspberry Pi model $\mathrm{B}+$.

An ultrasonic sensor array was considered to detect objects surrounding a wheelchair. Powered wheelchair users provided their desired direction using binary switches. Python programming language was used to create a program that acquired users' direction and analyzed information provided from the ultrasonic sensor array. A program was installed on a Raspberry Pi. The Raspberry Pi and the electronic circuit converted users input from switches into digital logic levels. A compromise between the desires of a driver and distance to nearby obstacles was conducted. If there was no obstacle detected in the direction supplied by the user, the Raspberry Pi activated a set of specific relays that provided power to the wheelchair motor responsible for driving the wheelchair in the chosen direction. If an obstacle was detected in that direction, the Raspberry Pi switched-off power to the motor responsible for driving the wheelchair in that direction and avoided the obstacle. Detection range could be digitally tuned by the intelligent controller from $2 \mathrm{~cm}$ to $500 \mathrm{~cm}$. New courses were simulated before testing in the laboratory using ultrasonic sensor arrays, Fig. 1. 


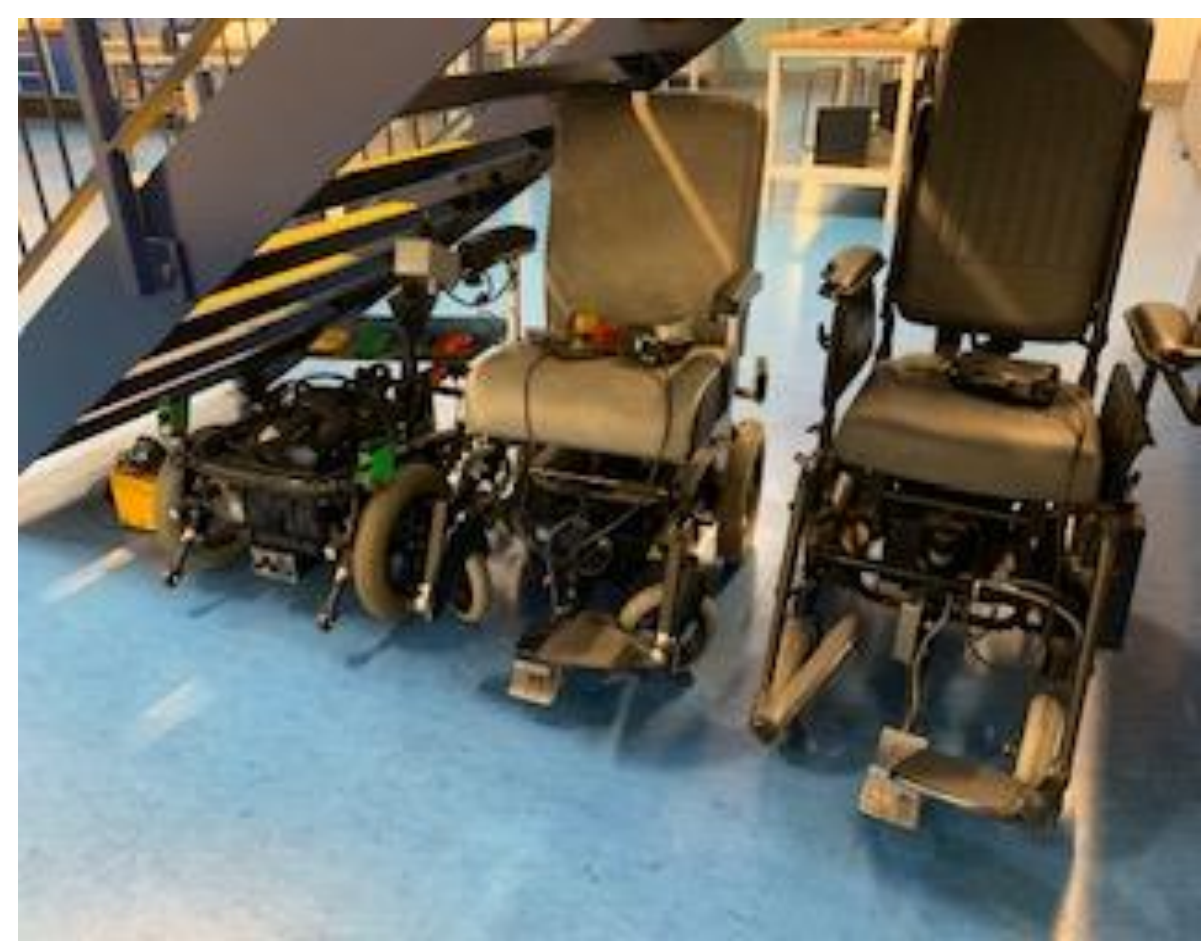

Fig. 1. Powered Wheelchairs considered for the research.

Many researchers considered sensors to assist wheelchair users with avoiding items safely [26]: infrared sensors [27], ultrasonics [28], and structured lighting [29]. Global methods cannot coordinated output when used inside buildings [30] and local systems provided more successful results: gyroscopes, odometers, tilt sensors and ultrasonics [31-33]. Cameras are becoming affordable but the processing of the data can be complicated. Computers are becoming affordable and more powerful [30], so cameras are often used. Despite that, a disabled driver can often provide the best data about what is required, but visual impairment or a disability may still reduce that ability [34].

In the work presented in this paper, ultrasonic sensors were considered due to their simplicity, robustness, and low cost [32]. Section 2 briefly explains the sensors, Section 3 presents the intelligent controller and $\mathrm{HCI}$ created to interface to the sensors. Section 4 presents results and Section 5 presents discussion and conclusions.

\section{Sensors}

Sensors considered in this research were the same as those used in [35]. Sensors were placed under footrests [36]. The distance from a detected object was determined by measuring the flight time required for a pulse to be sent and reflected back to a receiver(s) [37]. Fig. 2 shows the envelop of the ultrasonic sensor and a potential grid that can be created. 


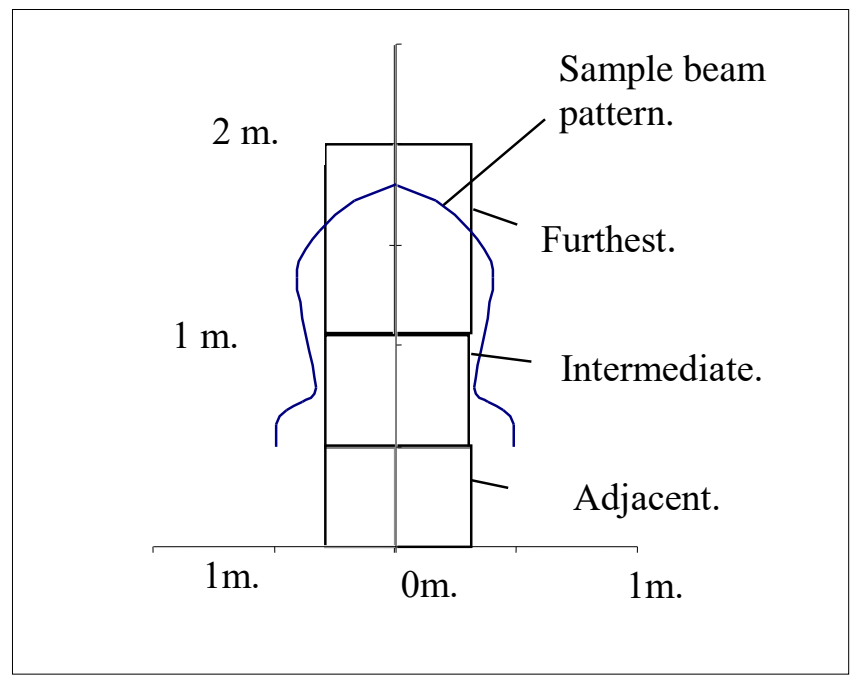

Fig. 2. The envelope for the ultrasonic sensor.

If no object was detected, then range could be digitally until an object was detected. Warnings could then be generated regarding obstructions ahead.

HC SR04 ultrasonic sensors were studied and tested by considering different objects to create polar plots. The physical architecture of these sensors prevented them from suffering from side lobe interference. Objects with different shapes and sizes were considered. The sensors detected small cylindrical objects with diameters less than 0.65 $\mathrm{cm} 15 \mathrm{~cm}$ away to larger objects 5 meters away. Figs. 3-6 show polar plots for different sensors detecting different objects at different distances.

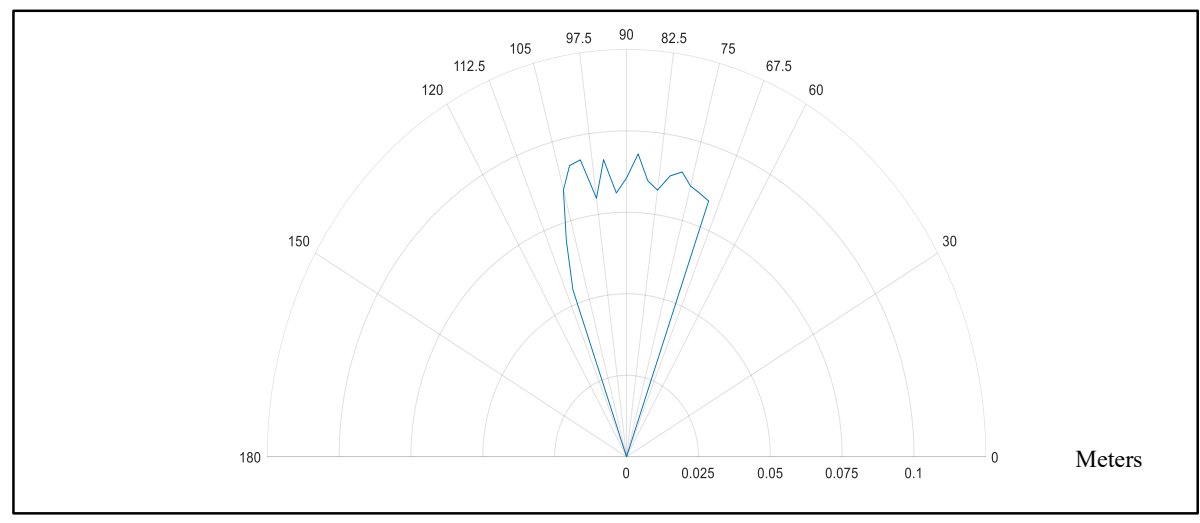

Fig. 3. Polar plot for HC SR04 ultrasonic sensor detecting $0.639 \mathrm{~cm}$ cylindrical metal object. 


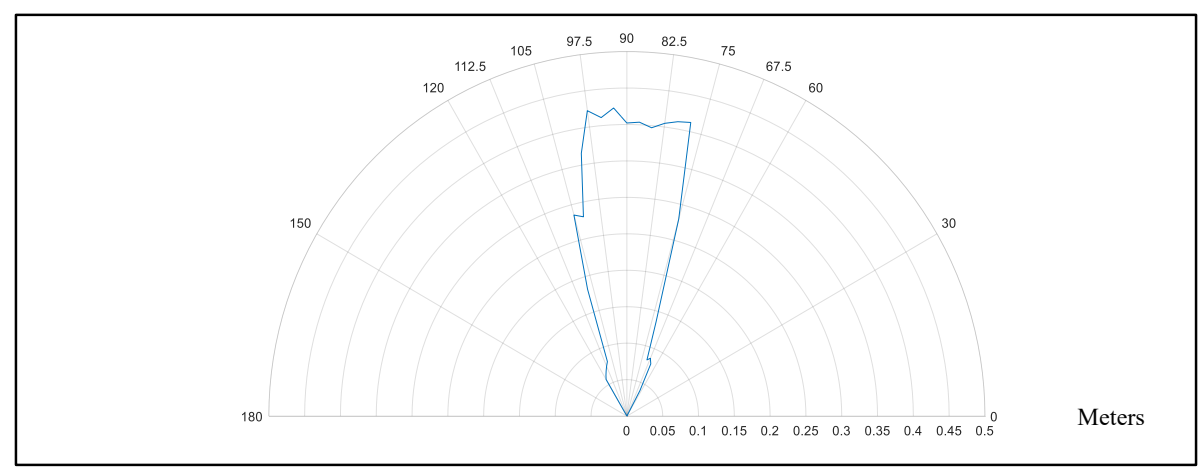

Fig. 4. Polar plot for HC SR04 ultrasonic sensor detecting $2.188 \mathrm{~cm}$ cylindrical metal object.

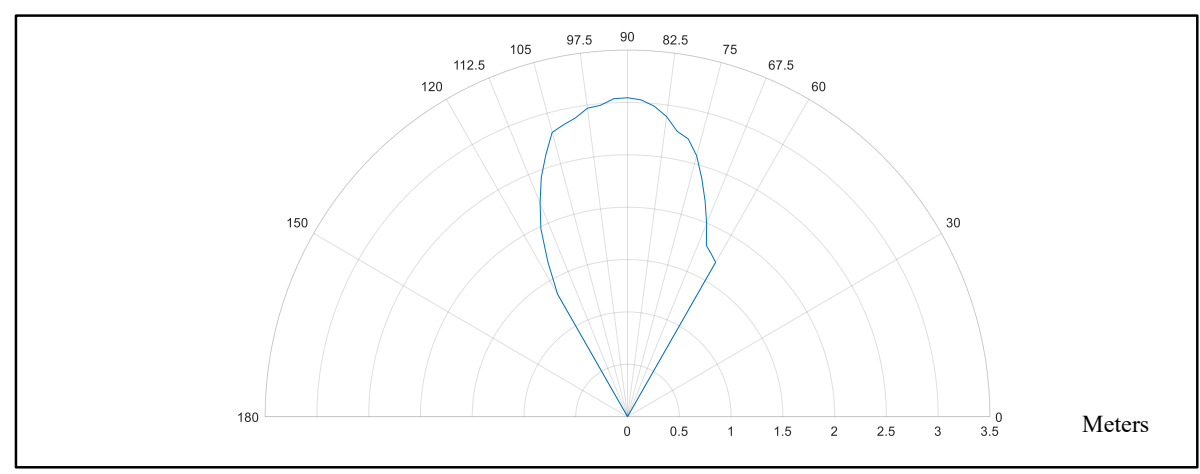

Fig. 5. Polar plot for HC SR04 ultrasonic sensor detecting $(4 \mathrm{X} 8) \mathrm{cm}$ rectangular plastic object.

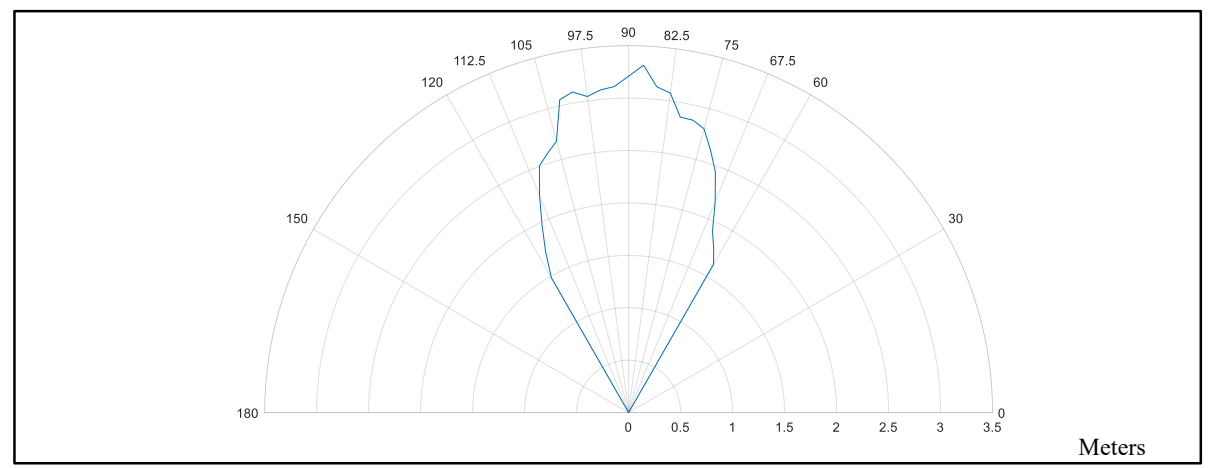

Fig. 6. Polar plot for HC SR04 ultrasonic sensor detecting (5.5X8.2) $\mathrm{cm}$ rectangular plastic object.

An architecture of five ultrasonic sensors was considered to produce an array, the ultrasonic sensors array was used to assess the wheelchair surroundings. Five HC SR04 ultrasonic sensors were used ( 3 were mounted to the front, 1 to the right and 1 to the left). The sensors provided distance to the nearest object in their detection zone. Python 
programming language was used to create a program that stopped a wheelchair from moving in the direction of an object if it was detected at a close distance. The range could be digitally tuned in the program from $2 \mathrm{~cm}$ to $500 \mathrm{~cm}$.

\section{Intelligent Controller and Human Computer Interface (HCI)}

An electronic circuit was created to digitise the output of the users' switches. A Raspberry Pi microcomputer was introduced between the users' switches and the wheelchair motors. The Raspberry Pi could be used as an intelligent controller and interface to any type of input device such as a joystick, chin, blow or head switch, or an EEG helmet.

User switches were connected the Raspberry Pi using a 9 pin D-connector. The following stages were used to digitize the output from the users' switches in order to create the intelligent controller and $\mathrm{HCI}$ :

1. Voltage drop $(12 \mathrm{~V} \rightarrow 5 \mathrm{~V})$ : The switches operated two $12 \mathrm{~V}$ DC motors. Raspberry Pi operated at 5V DC. The output from the input switches was reduced to 5V DC using CD4050BE Hex Buffer.

2. Python program 1: A program was created to acquire the output from switches.

3. Common collector circuit: Common collector circuits were created to operate the 5V DC relays. Fig. 7 shows the circuit diagram of the common collector circuit used. Fig. 8 shows a prototype of the circuits used.

4. Python program 2: Controls the wheelchair and provides intelligent HCI.

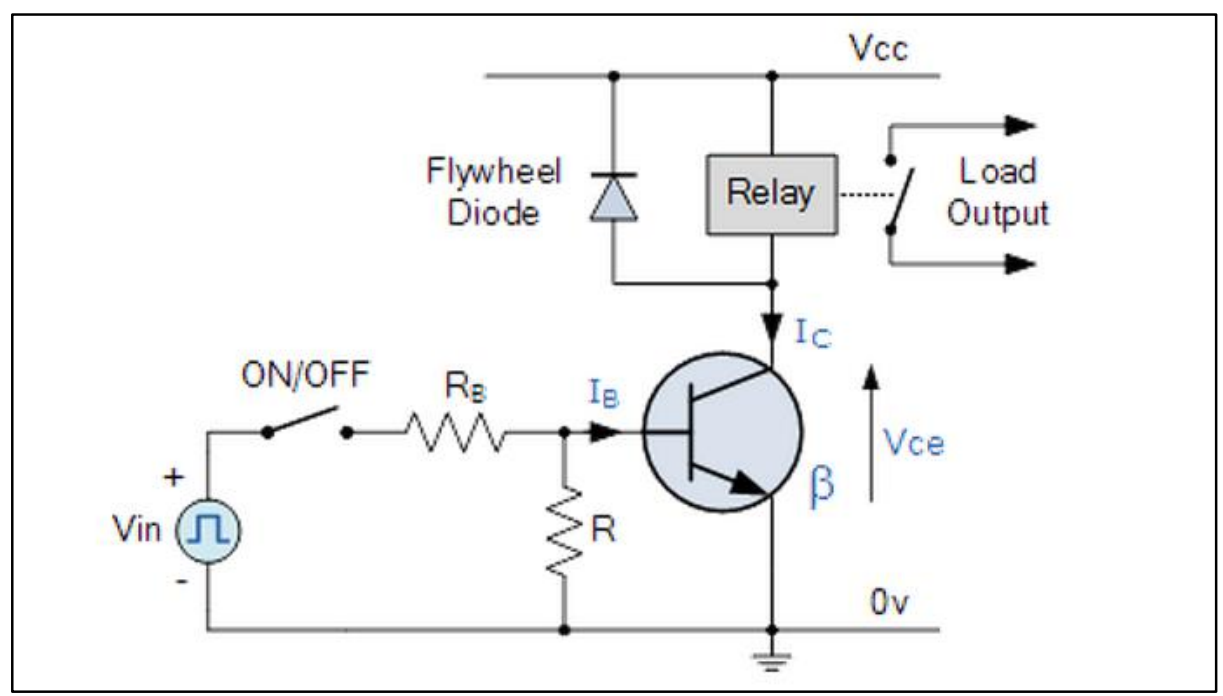

Fig. 7. Common Collector circuit diagram. 


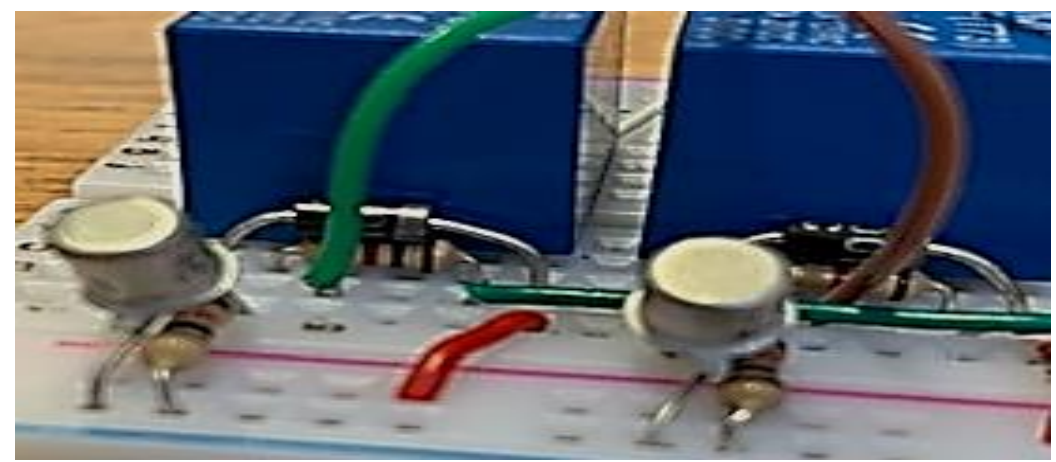

Fig. 8. Common Collector circuit considered for the new system.

A flywheel diode was used to provide a path for the current when the coil was switched off, this would prevent a voltage spike from occurring and damaging switch contacts or destroying switching transistors.

The architecture described in this section successfully interfaced input switches and intelligently controlled the operation of the switches to accurately transfer the desires of the wheelchair users to the wheelchair motors. The input from the switches (representing the wishes of the user) were passed on to a basic intelligent system contained within the Raspberry Pi. The software is written in Python. The information form the switches will then be combined with the input from the sensors to produce a new speed and direction. The new trajectory will be safer and avoid collision. A simple system has been created now to test scenarios and to gauge what might be possible. Once testing of the basic system is completed then more and more intelligence will be added.

\section{$4 \quad$ Results}

The work presented in this paper considered an ultrasonic sensor array to assess the surrounding of a powered wheelchair, digitized the output of users' switches and helped wheelchair users in driving their wheelchairs safely.

A driver provided a desired direction by pressing a switch, which triggered a specific $5 \mathrm{~V}$ DC relay that controlled the power supplied to the wheelchair motor responsible to drive the wheelchair in the desired direction.

Binary switches were used as an interface between a disabled user and their wheelchair to adjust bearing and speed. The digitization of the output of the binary switches combined with data from a sensor system could enhance driving by decreasing the number of collisions. Human drivers often use their own skill to drive their powered wheelchair, but sensors delivered a more repeatable and accurate reading, and could cover for any lack of ability or awareness. The new system blended human driving skill with autonomy and then involvement from the systems if necessary. When moving through complex or changing environment the sensors could provide better outcomes that could lead to safer decisions. 
Disabled wheelchair drivers could successfully drive their wheelchairs using their input device and the system avoided obstacles. The sensors ensured that the wheelchair was safe as it moved.

\section{Discussion and Conclusions}

The architecture presented in this paper is part of a larger research project concerned with improving mobility and enhancing the quality of life of disabled powered wheelchair users by increasing their self-confidence and reliance [1].

This paper presented a system that could interface any user input device to a powered wheelchair. The new systems might reduce the need for helpers and introduce more independence.

The electrical current that drove the motors was created from intelligently mixing the output from the sensor reading and the desired direction provided by a disabled driver. In an environment with few obstacles or if the obstacle(s) were far apart, then a driver would not need any support. If there were many obstacles in the way or there were obstructions close to the wheelchair, then the new sensor system was able to reduce the weight of the inputs from joysticks or switches to prevent a collision from happening.

The authors are currently studying ways to apply Machine Learning and other computationally inexpensive AI techniques to improve this research. Future work will consider the application of Machine Learning techniques and aim at collecting user data to predict driving patterns, assess driving skills progress and analyze ability factors denoting the ability of a user to operate their wheelchair. Specifically some decisionmaking algorithms will be investigated [38 - 40].

Results showed that the new system performed accurately. Systems and methods are clinically trialed at Chailey Heritage School as part of a larger ERSPC research project [1].

\section{Acknowledgment}

Research in this paper was funded by EPSRC grant EP/S005927/1 and supported by The Chailey Heritage Foundation and the University of Portsmouth.

\section{References}

1. Sanders, D. and Gegov, A.: Using artificial intelligence to share control of a powered-wheelchair between a wheelchair user and an intelligent sensor system, EPSRC Project 2019 - 2022 (2018).

2. Joshi, M. K., Gupta, M. V., Gosavi, M. M., \& Wagh, M. S. (2015). A multifunctional smart wheelchair. Int. J. Adv. Res. Electron. Commun. Eng., 4(5), 1281-1284. 
3. Leaman, J., \& La, H. M. (2017). A comprehensive review of smart wheelchairs: past, present, and future. IEEE Transactions on Human-Machine Systems, 47(4), 486-499.

4. Sanders, D. A.: Non-model-based control of a wheeled vehicle pulling two trailers to provide early powered mobility and driving experiences. IEEE Transactions on Neural Systems and Rehabilitation Engineering, 26,(1), 96 104, (2018).

5. Parhi, D. R. and Singh, M. K.: Rule-based hybrid neural network for navigation of a wheelchair, Proc. IMechE Part B: J. Engineering Manufacture, 224, 11103 - 1117 (2009).

6. Sanders, D. A. Gegov, A. and Ndzi, D.: Knowledge-based expert system using a set of rules to assist a tele-operated mobile robot, in Studies in Computational Intelligence, (eds) Y. Bi, S. Kapoor, and R. Bhatia, 2018, 751, Springer, pp. 371-392 (2018).

7. Sanders, D. A. et al.: Rule-based system to assist a tele-operator with driving a mobile robot. Lecture Notes in Networks and Systems, 16, Springer, pp. 599-615 (2018).

8. Haddad, M., Sanders, D., Gegov, A., Hassan Sayed, M., Huang, Y. and AlMosawi, M.: Combining multiple criteria decision making with vector manipulation to decide on the direction for a powered wheelchair. in $\mathrm{Y} \mathrm{Bi}, \mathrm{R}$ Bhatia \& S Kapoor (eds), Advances in Intelligent Systems and Computing, vol. 1037, Springer, pp. 680-693 (2019).

9. Haddad, M. and Sanders, D.: Selecting a best compromise direction for a powered wheelchair using PROMETHEE. IEEE Transactions on Neural Systems and Rehabilitation Engineering, 27(2), 228-235 (2019).

10. Haddad, M., Sanders, D., Tewkesbury, G., Gegov, A., Hassan Sayed, M. and Ikwan, FC.: Initial results from using Preference Ranking Organization METHods for Enrichment of Evaluations to help steer a powered wheelchair. in Y Bi, R Bhatia \& S Kapoor (eds), Advances in Intelligent Systems and Computing, vol. 1037, Springer, pp. 648-661 (2019).

11. Sanders, D., Tewkesbury, G., Parchizadeh, H., Robertson, JJ., Omoarebun, PO. and Malik, M.: Learning to drive with and without intelligent computer systems and sensors to assist. in K Arai, S Kapoor \& R Bhatia (eds), Advances in Intelligent Systems and Computing, vol. 868, Springer, pp. 1171-1181 (2019).

12. Sanders, D., Gegov, A., Haddad, M., Ikwan, F., Wiltshire, D. and Tan, YC.: A rule-based expert system to decide on direction and speed of a powered wheelchair. in K Arai, S Kapoor \& R Bhatia (eds), Advances in Intelligent Systems and Computing, vol. 868, Springer, pp. 822-838 (2019).

13. Haddad, M., Sanders, D., Bausch, N., Tewkesbury, G., Gegov, A. and Hassan Sayed, M.: Learning to make intelligent decisions using an Expert System for the intelligent selection of either PROMETHEE II or the Analytical Hierarchy Process. in K Arai, S Kapoor \& R Bhatia (eds), Advances in Intelligent Systems and Computing, vol. 868, Springer, pp. 1303-1316 (2019).

14. Sanders, D., Gegov, A., Tewkesbury, G. and Khusainov, R.: Sharing driving between a vehicle driver and a sensor system using trust-factors to set control 
gains. in K Arai, S Kapoor \& R Bhatia (eds), Advances in Intelligent Systems and Computing, vol. 868, Springer, pp. 1182-1195 (2019).

15. Sanders, D., Langner, M., Bausch, N., Huang, Y., Khaustov, SA. and Simandjuntak, S.: Improving human-machine interaction for a powered wheelchair driver by using variable-switches and sensors that reduce wheelchair-veer. in Y BI, R Bhatia \& S Kapoor (eds), Advances in Intelligent Systems and Computing, vol. 1038, Springer, pp. 1173-1191 (2019).

16. Okonor, OM., Gegov, A., Adda, M., Sanders, D., Haddad, M. and Tewkesbury, G.: Intelligent approach to minimizing power consumption in a cloud-based system collecting sensor data and monitoring the status of powered wheelchairs. in Y Bi, R Bhatia \& S Kapoor (eds), Advances in Intelligent Systems and Computing, vol. 1037, Springer, pp. 694-710 (2019).

17. Sanders, D., Okonor, OM., Langner, M., Hassan Sayed, M., Khaustov, SA. and Omoarebun, PO.; Using a simple expert system to assist a powered wheelchair user. in Y Bi, R Bhatia \& S Kapoor (eds), Advances in Intelligent Systems and Computing, vol. 1037, Springer, pp. 662-379 (2019).

18. Parhi D. R. et al.: "The stable and precise motion control for multiple wheelchairs. Appl. Soft Comput, 9(2), 477 - 487 (2009).

19. Nguyen, V. et al.: Strategies for Human - Machine Interface in an Intelligent Wheelchair, 35th Annual Int Conf of IEEE- (EMBC), Osaka, JAPAN. Book Series: IEEE Engineering in Medicine \& Biology Society Conf Proceedings, pp. 3638-3641 (2013).

20. Tewkesbury, G., Sanders, D., Haddad, M., Bausch, N., Gegov, A. and Okonor, OM.: Task programming methodology for powered wheelchairs. Advances in Intelligent Systems and Computing, Springer, IEEE SAI Intelligent Systems Conference 2019, London, United Kingdom, Vol. 1 pp. $711-720$ (2019).

21. Sanders, D., Wang, Q., Bausch, N., Huang, Y., Khaustov, SA. and Popov, I.: A method to produce minimal real time geometric representations of moving obstacles. in K Arai, S Kapoor \& R Bhatia (eds), Advances in Intelligent Systems and Computing, vol. 868, Springer, pp. 881-892 (2019).

22. Bausch, N., Shilling, P., Sanders, D., Haddad, M., Okonor, OM. and Tewkesbury, G.: Indoor location and collision feedback for a powered wheelchair system using machine learning. Advances in Intelligent Systems and Computing, Springer, IEEE SAI Intelligent Systems Conference 2019, London, United Kingdom, Vol. 1 pp. 721-739 (2019).

23. Sanders, D. A. et al.: Results from investigating powered wheelchair users learning to drive with varying levels of sensor support, in Proc SAI Intelligent System, London, U.K. pp 241-245 (2017).

24. Song, K. T. and Chen, C. C.: Application of asymmetric mapping for wheelchair navigation using ultrasonic sensors. J. Intell. Wheelchair Syst., 17(3), $243-264$ (1996).

25. Sachdeva, P. and Katchii, S.: A Review Paper on Raspberry Pi. International Journal of Current Engineering and Technology, 4(6), 3818 - 3819 (2014).

26. Sanders, D., Langner, M. and Tewkesbury, G.: Improving wheelchair- driving using a sensor system to control wheelchair-veer and variable-switches as an alternative to digital-switches or joysticks. Ind Rob: An int' jnl, 37(2), 151167 (2010). 
27. Lee, S.: Use of infrared light reflecting landmarks for localization. Ind Rob: An int' jnl, 36(2), 138-145 (2009).

28. Sanders, D. and Stott, I.: A new prototype intelligent mobility system to assist powered wheelchair users. Ind Rob, 26(6), 466-475 (2009).

29. Larsson, J., Broxvall, M. and Saffiotti, A.: Laser-based corridor detection for reactive Navigation. Ind Rob: An int' jnl, 35(1), 69-79 (2008).

30. Milanes, V., Naranjo, J. and Gonzalez, C.: Autonomous vehicle based in cooperative GPS and inertial systems. Robotica, 26, 627-633 (2008).

31. Sanders, D. A.: Controlling the direction of walkie type forklifts and pallet jacks on sloping ground. Assem. Autom., 28(4), 317-324 (2008).

32. Sanders, D.: Recognizing shipbuilding parts using artificial neural networks and Fourier descriptors. Proceedings of the Institution of Mechanical Engineers - Part B- Journal of Engineering Manufacture, 223(3), 337-342 (2009).

33. Chang, Y. C. and Yamamoto, Y.: On-line path planning strategy integrated with collision and dead-lock avoidance schemes for wheeled wheelchair in indoor environments. Ind Rob: An int' jnl, 35(5), 421-434 (2008).

34. Sanders, D.: Comparing speed to complete progressively more difficult mobile robot paths between human tele-operators and humans with sensorsystems to assist. Assem. Autom., 29(3), 230-248 (2009).

35. Sanders, D. A. and Bausch, N.: Improving Steering of a Powered Wheelchair Using an Expert System to Interpret Hand Tremor. Proc of Intelligent Wheelchairics and Applications (Icira 2015), Pt Ii, vol. 9245, pp. 460-471, (2015).

36. Sanders, D. A.: Using self-reliance factors to decide how to share control between human powered wheelchair drivers and ultrasonic sensors. IEEE Transactions on Neural Systems and Rehabilitation Engineering, 25(8), 12211229 (2017)

37. Sanders, D. A. et al.: Tele-operator performance and their perception of system time lags when completing mobile robot tasks, Proc 9th Int Conf on Human Systems Interaction, pp. 236-242 (2016).

38. Haddad, M., Sanders, D. and Bausch, N.: Selecting a robust decision making method to evaluate employee performance. International Journal of Management and Decision Making, 18(4), pp. 333-351 (2019).

39. Haddad, M., Sanders, D. and Tewkesbury, G.: Selecting a discrete Multiple Criteria Decision Making method to decide on a corporate relocation. Archives of Business Research,7(5), pp. 48-67 (2019).

40. Sanders, D., Robinson, DC., Hassan Sayed, M., Haddad, M., Gegov, A. and Ahmed, N.: Making decisions about saving energy in compressed air systems using Ambient Intelligence and Artificial Intelligence. in K Arai, S Kapoor \& R Bhatia (eds), Advances in Intelligent Systems and Computing, vol. 869, Springer, pp. 1229-1236 (2019). 